\title{
Liturgical chant bibliography 30
}

\author{
RAQUEL ROJO CARRILLO and MARIE WINKELMÜLLER-URECHIA
}

After more than a year of challenges caused by the COVID-19 pandemic, we hope that you and your loved ones have managed to remain healthy, active and as happy as possible. The delay of several publications has resulted in a LCB slightly shorter than those produced in the years ante coronam. The list of publications included in this year's LCB shows that our field is thriving despite the various challenges. We are committed to fill any possible gaps in upcoming LCBs, hoping that the numbers of COVID-19 cases decrease and allow restrictions to ease.

As occurred with LCB 29, this year's LCB was produced under the challenges linked with the pandemic. We were faced again with libraries either entirely closed or with restricted access. In times like this, we are even more grateful for the help and generosity of colleagues and librarians who very kindly provided us with lists of the most recent publications in the field, and even with pictures of the indexes of publications that we would have otherwise been unable to access. We thank them very much for their effort and collaboration.

This year brought two editions of importance, the first being a Liber ordinarium from the diocese of Freising near Munich, continuing the long series of F.K. Prassl's team. The other is a critical edition of the Jistebnice Kancionál by Hana Vlhová-Wörner. The Slovakian team published a series of publications on the Missale Notatum Bratislava, Ústredna knižnica Slovenskej akadémie vied Rkp. zv. 387.

Fragmentary sources feature as an important topic this year, with twelve publications related to or building on the worldwide Fragmentarium Project, and with the development of a fragments' database as well (30005, 30017k, 30018e, 30022d, $30028,30032,30045 a, 30045 b, 30051,30082,30086,30096)$. These achievements have a great potential of prompting future investigations on fragments, and we hope to see new titles related to this topic in future LCBs.

Also worth mentioning is the development of the Catalogue des manuscrits notés en neumes français de la Bibliothèque nationale de France (30095), a database that gathers, describes and aids the analyses of notated manuscripts from this important library. This database aims to help researchers in search of notational influences and concordances.

We wish you, dear reader, the most productive time despite the pandemic. We will hopefully meet again soon. For any question, suggestion, and/or bibliographic information, you can reach us through the email address: liturgchantbiblio@gmail.com.We thank you in advance for your help.

\section{Additions}

27/28003R. Luisa NARDINI, Speculum, 95/4 (2020), 1178-9. 
27/28016R. Franz Karl PRASSL, Beiträge zur Gregorianik, 68 (2019), 89-92.

27/28024R. Maria Incoronata COLANTUONO, Medievalia. Revista d'Estudis Medievals, 23/2 (2020), 135-7. - Daniel SAULNIER, Revue de musicologie, 106/1 (2020), 237-41.

27/28027R. Cynthia J. CYRUS, Speculum, 96/2, 559-61.

29003R. Laura ALBIERO, Fragmentology, 3 (2020), 149-54.

29014R. Andreas JANKE, Die Musikforschung, 73/2 (2020), 156-7.

29017R. Marie WINKELMÜLLER-URECHIA, Die Musikforschung, 74/1 (2021), 60-1.

\section{Editions and facsimile editions}

30001. Michael BERNHARD, Klaus-Jürgen SACHS, Musiklehre zwischen Mittelalter und Humanismus: das Studienkonvolut des Stephan Roth (Zwickau, Ratsschulbibliothek 24.10.26), Veröffentlichungen des Staatlichen Instituts für Musikforschung 24/Studien zur Geschichte der Musiktheorie 14 (Hildesheim: Olms, 2019).

Review. Joseph DYER, Plainsong E Medieval Music, 29/2 (2020), 177-80.

30002. Gionata BRUSA, Der Liber Ordinarius der Diözese Freising. Eine textkritische Edition des mittelalterlichen Regelbuchs der Diözese Freising, Codices Manuscripti \& Impressi. Supplementum 19 (Purkersdorf: Hollinek, 2020).

30003. Hana VLHOVÁ-WÖRNER, et al., The Jistebnice Kancionál. Prague, National Museum Library, II C 7. Critical Edition, Monumenta Liturgica Bohemica (Brno: Luboš Marek, 2019).

\section{Books, reprints}

30004. Rastislav ADAMKO, Janka BEDNÁRIKOVÁ, Zuzana ZAHRADNIKOVÁ, Eva VESELOVSKÁ, Rastislav LUTZ, Das Missale notatum Rkp. zv. 387 - eine skandinavische Handschrift in der Slowakei. Missale notation Rkp. zv. 387 - skandinavski rokopis na Slovaškem, https://revije.ff.uni-lj.si/MuzikoloskiZbornik/article/view/9389.

30005. Veronika GARAJOVÁ, Catalogus fragmentorum cum notis musicis medii aevi e civitate Trenchini. Reviewers Janka Bednáriková, Rastislav Adamko, Catalogus fragmentorum cum notis musicis medii aevi in Slovakia 6 (Bratislava: Institute of Musicology of the Slovak Academy of Sciences, 2020).

30006. Gernot GRUBER, Kulturgeschichte der europäischen Musik. Von den Anfängen bis zur Gegenwart (Kassel: Bärenreiter/Berlin: Metzler, 2020).

30007. Hildegard HERRMANN-SCHNEIDER, Wo die Engel musizieren: Musik im Stift Stams (Brixen: Verlag A. Weger, 2020).

30008. Christoph HÖNERLAGE, Centonisation als Interpretation. Formelfunktionen und Wort-Ton-Verhältnis in den Gradualien des V. Modus (St Ottilien: EOS, 2020).

30009. Marie T. LEVEY, The Place of the Kyriale - the Ordinary of the Mass - in Catholic History, Liturgy and Music (Burwood, New South Wales: Trustees of the Sisters of St Joseph, 2020).

30010. Richard MAILÄNDER, Basiswissen Kirchenmusik, Vol. 1: Theologie-Liturgiegesang, 3. aktualisierte und erweiterte Auflage (Stuttgart: Carus-Verlag, 2020). 
30011. Rebecca MALOY, Songs of Sacrifice: Chant, Identity, and Christian Formation in Early Medieval Iberia (New York: Oxford University Press, 2020).

30012. Stefan MORENT, Die Musik der Antike und des Mittelalters, Epochen der Musikgeschichte 1 (Laaber: Laaber Verlag, 2020).

30013. Raquel ROJO CARRILLO, Text, Liturgy, and Music in the Hispanic Rite: The Vespertinus Genre (New York: Oxford University Press, 2021).

30014. Stefania RONCROFFI, I manoscritti di canto liturgico della Biblioteca dell' Archiginnasio e del Museo della Musica di Bologna, con una sezione di Milena BASILI sui Frammenti musicali del Museo della Musica, Biblioteca de 'L'Archiginnasio', Serie III, n. 14 (Bologna: Comune di Bologna, 2021).

\section{Congress proceedings}

30015. France et Angleterre. Manuscrits médiévaux entre 700 et 1200, ed. Charlotte DENOËL, Francesco SIRI, Bibliologia 57 (Turnhout: Brepols, 2020):

(a) Laura ALBIERO, ‘Interférences liturgiques entre France et Angleterre', 281-9.

30016. Il tempo delle comunità monastiche nell'alto Medioevo. Atti del Convegno internazionale di Studio, Roma-Subiaco, 9-11 giugno 2017, ed. Letizia ERMINI PANI, De Re Monastica 6 (Spoleto: CISAM, 2020):

(a) Laura ALBIERO, 'Manoscritti liturgici monastici dell'Italia meridionale: tipologie e funzioni', 149-61.

30017. IMS Study Group Cantus Planus: Papers read at the XVII meeting, Venice, Italy, 28 July-1 August 2014, ed. James BORDERS (Venice: Edizioni Fondazione Levi, 2020):

(a) Davide CROFF, 'Presentation', ix-x. (b) Roberto CALABRETTO, Luisa ZANONCELLI, 'Preface', xi-xii. (c) James BORDERS, 'Foreword to the Meeting', xiii. (d) Nausica MORANDI, 'Opening address', xiv. (e) Andreas PFISTERER, 'Zur Bedeutung von Oxeia/Acutus/Virga in den griechischen und lateinischen Neumenschriften', 3-8. (f) Laura ALBIERO, 'From France to northern Italy: specific features in "Comasca" notation', 9-18. (g) Elsa DE LUCA, 'A methodology for studying Old Hispanic notation: some preliminary thoughts', 19-40. (h) David CATALUNYA, "The "codification" of new Latin song in early twelfth century: codicological insights into F-Pn fonds latin 1139', 43-7. (i) Marco GOZZI, 'Manuscripts in Cortona: fragments and liturgical books in the Archivio storico diocesano', 49-60. (j) Karin Strinnholm LAGERGREN, 'The Birgittine Abbey of Maria Refugie: Five hundred years of manuscript production', 61-71. (k) Santiago RUIZ TORRES, Juan Pablo RUBIO SADIA, 'Liturgical fragments of the diocese of Sigüenza (eleventh-sixteenth centuries)', 73-82. (1) Rebekka SANDMEIER, 'Imposing European culture on the Cape Colony: medieval manuscripts in the Grey collection', 83-93. (m) James BORDERS, 'A northern Italian intermediary between Avignon and Rome? Oxford, Bodleian Library, MS. Canonici Liturgical 375 and the chants of the 1485 Pontificale Romanum', 95-106. (n) Jurij SNOJ, 'The antiphoner of Izola', 107-16. (o) Réka MIKLÓS, 'Der Seckauer Liber ordinarius von ca. 1595 (A-Gu 1566) als letztes Dokument der mittelalterlichen Salzburger-Seckauer Liturgie und Musik', 117-34. (p) Andreas HAUG, 'Towards a semiotically informed transcription practice', 137-42. (q) Konstantin VOIGT, 'Reconstructing acts of writing. Editorial consequences of writing scenarios assumed for the versus Annus novus in Paris 1139', 143-50. (r) Elaine Stratton HILD, 'Working realities of the New Philology: considering the potential of 
technology in editing medieval texts', 151-4. (s) Debra LACOSTE, Jan KOLÁČEK, 'Cantus for office and mass: building an online network of chant databases', 157-62. (t) Kate HELSEN, Inga BEHRENDT, Jennifer BAIN, 'A new way to see neumes: the optical neume recognition project in context and in action', 163-7. (u) Gábor KISS, 'Late flourishing of the alleluia repertory in central Europe', 171-85. (v) Giulia GABRIELLI, 'Tropes in cantus planus sources from South Tyrol', 187-96. (w) Marit Johanne HØYE, 'Kyrie chants in manuscripts from the German-speaking area', 197-212. (x) Sarah Ann LONG, 'Mensurally notated mass ordinaries from Cambrai and Tournai', 213-23. (y) Hana VLHOVÁ-WÖRNER, 'Agnus pairing and disappearing: a contribution to the late chant tradition in Bohemia', 225-38. (z) Morné BEZUIDENHOUT, 'In search of black swans: a computer-assisted approach to interval-pattern recognition', 241-52. (aa) Dirk VAN BETTERAY, 'Textinterpretation und Formelkomposition. Heiligenoffizien im Codex Hartker', 253-65. (ab) Roman HANKELN, 'The articulation of direct speech in responsories', 267-78. (ac) Danette BRINK, 'Simeon: the long journey to Trier and Sainthood', 279-89. (ad) Raquel ROJO CARRILLO, 'The vespertini as witnesses to the transmission of Old Hispanic chant in traditions A and B', 293-309. (ae) Hanna ZÜHLKE, 'Hirsau in Norditalien: Zur liturgischen Musikpraxis des Benediktinerklosters Moggio im zwölften und dreizehnten Jahrhundert', 311-21. (af) Nausica MORANDI, 'The Office for Saint Cecilia in musico-liturgical manuscripts from the Pontificia Biblioteca Antoniana of Padua', 323-36. (ag) Stefania RONCROFFI, 'Antichi testimoni dell'ufficio di san Prospero e tradizione nei codici reggiani', 337-44. (ah) Diego TOIGO, 'Il canto del Passio presso alcuni ordini religiosi in fonti italiane tardive', 345-59. (ai) Jean-François GOUDESENNE, 'Apostolicité et exports liturgiques en France méridionale, Gaule cisalpine et Italie non romaine (835-1150)', 359-76. (aj) Océane BOUDEAU, 'Le sanctoral de la cathédrale de Sens', 377-88. (ak) Tova LEIGH-CHOATE, 'The weekly commemorative office: a twelfth-century witness from Saint-Denis in context', 389-400. (al) Melanie BATOFF, 'The Visitatio sepulchri as a Gospel Harmony in medieval Germany', 401-12. (am) Alison ALTSTATT, 'Singing the saints in Medieval Eichstätt: the case of Wolfhard of Herrieden's office for Saint Walburga', 413-24. (an) Michelle URBERG, 'Learning gender roles from the Paschal and Mariological processions of the brothers and sisters at Vadstena Abbey', 425-36. (ao) Manuel Pedro FERREIRA, 'Venetian influence in fifteenth-century Portugal', 43750. (ap) Orsolya CSOMÓ, 'The feasts of the Holy Virgin in the liturgy of the mass in Zagreb Cathedral and its Mediterranean context', 451-61. (aq) Thomas Forrest KELLY, Katarina LIVLJANIĆ, 'Ferial office in the Montecassino antiphoner and in Beneventan sources of the eleventh and twelfth centuries', 465-79. (ar) Luisa NARDINI, 'The masses for the Dead in Beneventan manuscripts: Issues of formulary organization and chant manipulation', 481-94. (as) Matthew PEATTIE, 'Graphic difference and the interpretation of the climacus in Beneventan notation', 495-515. (at) Bibiana GATTOZZI, 'The hymn in Beneventan manuscripts', 517-29. (au) Svetlana KUJUMDZIEVA, 'The tropologion Vaticanus Graecus 771', 533-48. (av) Silvia TESSARI, 'The Byzantine musical manuscripts of the Veneto region: overview of a current research project. One example (Bassan. gr. 34B19)', 549-61. (aw) Gerda WOLFRAM, 'The Byzantine tradition of the great doxology', 563-8. (ax) Nina-Maria WANEK, 'O quando in cruce - "O $\tau \epsilon \tau \omega$

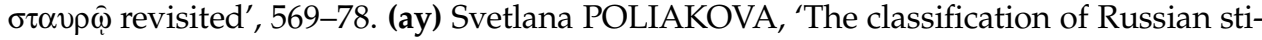
cheraria: a preliminary case study', 579-84. (az) Elizabeth J. MARKHAM, 'A schematic musical form for singing Buddhist hymns in the "bilingual" literary culture of early Japan', 585-91.

30018. Religion - Musik - Macht. Musikalische Dimensionen einer ästhetischen Theologie, ed. Wolfgang W. MÜLLER, Franc WAGNER, TeNor - Text und Normativität 8 (Basel: Schwabe Verlag, 2021): 
(a) Franc WAGNER, 'Zur Interdependenz von Wort und Ton in der Musik seit der Antike', 13-36. (b) Franz Karl PRASSL, "'Dass ich den text behandelt habe, wie er noch wenig behandelt worden". Paradigmen für eine Musik der Kirche', 141-77. (c) Konstantinos NIKOLAKOPOULOS, 'Grundlegende Merkmale der byzantinischen Musik und ihr Einsatz in den orthodoxen Gottesdiensten', 205-16. (d) Stefan KLARER, 'Singende Mönche - singende Gemeinde? Gregorianische Aufführungspraxis in der Schweiz', 375-96. (e) Stefan MORENT, 'Europäische und regionale Musikkultur im Kontext des Konstanzer Konzils. Bericht zur Erforschung liturgischer Musikfragmente des Stadtarchivs Konstanz', 397-423.

30019. Beiträge zur Gregorianik, 69 (2020):

\section{Chant journals}

(a) Franco ACKERMANS, Luigi AGUSTONI ( + ), Inga BEHRENDT, Rupert FISCHER ( + ), Johannes Berchmans GÖSCHL, Christoph HÖNERLAGE, Liobgid KOCH, Josef KOHLHÄUFL, Stefan METZ, Daniel RÖSLER, Heinrich RUMPHORST, Anton STINGL jun., Stephan ZIPPE, 'Vorschläge zur Restitution von Melodien des Graduale Romanum, Teil 45', 9-36. (b) Anton STINGL jun., 'Das Offertorium Felix namque es', 37-50. (c) Godehard JOPPICH, 'Die Liqueszenz - Eine semiologische Studie im Codex Hartker St. Gallen 390/391 - Teil 2 (Nachdruck)', 51-98. (d) Heinrich RUMPHORST, 'Die Gesangstexte der Offertorien und ihrer Verse im Vergleich mit ihren Schriftquellen - mit deutscher Übersetzung - Teil $3(135,1-5)^{\prime}, 121-40$.

30020. Beiträge zur Gregorianik, 70 (2020):

(a) Franco ACKERMANS, Luigi AGUSTONI (†), Inga BEHRENDT, Rupert FISCHER (†), Johannes Berchmans GÖSCHL, Christoph HÖNERLAGE, Stephan KLARER, Josef KOHLHÄUFL, Stefan METZ, Daniel RÖSLER, Heinrich RUMPHORST, Anton STINGL jun., Stephan ZIPPE, 'Vorschläge zur Restitution von Melodien des Graduale Romanum, Teil 46', 9-22. (b) Franz Karl PRASSL, 'Der Codex Hartker (CH-SGs 390/ 391): Rhetorik und Rhythmische Artikulation als Ausdruck liturgischer Theologie', 23-42. (c) Iun NISHIWAKI, 'Amalar und die liturgischen Gesänge des Weihnachtsfestkreises', 43-8. (d) Inga BEHRENDT, 'Die "feinen Fliegenfüße der St. Galler und Einsiedelner Neumen des 9.-10. Jahrhunderts": Hugo Riemanns Verständnis des Choralrhythmus Anfang des 20. Jahrhunderts', 49-64.

30021. Études grégoriennes, 47 (2020):

(a) Joseph DYER, 'Theories of origin and survival of Old Roman chant', 1-24. (b) Eduardo Henrik AUBERT, 'Sur les traces d'une culture musicale: Notations musicales occasionnelles dans les manuscrits de Cluny jusqu'au XII ${ }^{\mathrm{e}}$ siècle', 25-59. (c) Océane BOUDEAU, 'Un office médiéval en l'honneur de saint Déodat', 61-94. (d) Cillian LONG, 'The theory and practice of plainchant accompaniment (part one). Deluded antiquarianism to c. 1860: the shock of the old', 95-139. (e) Xavier PERRIN o.s.b., "'Lingua mea calamus scribæ velociter scribentis" ou de la spiritualité des neumes', 141-53.

30022. Studi gregoriani, 33 (2017): Atti del Convegno di Studi Il Canto Ambrosiano, Convegno internazionale di Studi Milano-Lugano, 8-10 settembre 2017, ed. Angelo RUSCONI, Guido MILANESE, Vol. 1:

(a) Angelo RUSCONI, Guido MILANESE, 'Introduzione', 9-12. (b) Claudio MAGNOLI, 'Premessa', 13-16. (c) Terence BAILEY, 'An Introduction to the Ambrosian Processional Antiphons', 17-26. (d) Jacopo Maria CALLONI, ‘Un Frammento 
sconosciuto di antifonario ambrosiano', 27-62. (e) Matteo GARZETTI, 'Un melisma del versus Dedisti letitiam', 63-86. (f) Marco GOZZI, 'L'Antifonario ambrosiano trecentesco della Biblioteca musicale Laurence Feininger di Trento (Trento, Biblioteca del Castello di Buonconsiglio, Fondo Feininger, ms. FC 74)', 87-116. (g) Thomas F. KELLY, 'The Earliest Notations of Ambrosian Chant', 117-22. (h) Angelo RUSCONI, 'Il Credo nella messa ambrosiana', 123-96.

30023. Studi gregoriani, 34 (2018): Atti del Convegno di Studi Il Canto Ambrosiano, Convegno internazionale di Studi Milano-Lugano, 8-10 settembre 2017, ed. Angelo RUSCONI, Guido MILANESE, Vol. 2:

(a) Giulia GABRIELLI, 'Gli inni nelle edizioni ambrosiane con musical del Seicento', 9-36. (b) Gionata BRUSA, 'Influssi ambrosiani nelle tradizione liturgico-musicale di Vercelli', 37-74. (c) Luca RICOSSA, 'Modalità, struttura e retorica nel canto ambrosiano', 75-86. (d) Francesco ANDREONI, 'Qualche considerazione e alcuni esempi di declinazione di ambrosiano rustico', 87-106. (e) Jakub KUBIENIEC, 'Longing for Milan? Ambrosian chants in a Breviary from Kraków', 107-22.

30024. Studi gregoriani, 35 (2019): Atti del Convegno di Studi Il Canto Ambrosiano, Convegno internazionale di Studi Milano-Lugano, 8-10 settembre 2017, ed. Angelo RUSCONI, Guido MILANESE, Vol. 3:

(a) Laila GAGLIANO, Stefano Maria MALASPINA, 'Strumenti e fonti per lo studio del canto ambrosiano nella Biblioteca del Capitolo Metropolitano di Milano', 9-20. (b) Norberto VALLI, 'Il responsorio Tenebrae e le antifone per l'adorazione della Croce nella liturgia ambrosiana del Venerdì Santo', 21-34. (c) Fabio STIRPE, 'Antifone ambrosiane su un processionale beneventano del secolo XI. Studio comparato del processionale in notazione beneventana contenuto nel manoscritto Vaticanus Reginensis Latinus 334 con i repertori ambrosiano e gregoriano', 35-126.

\section{Collections of essays, dictionaries}

30025. A Companion to the English Dominican Province: From Its Beginnings to the Reformation, ed. Eleanor J. GIRAUD, J. Cornelia LINDE, Brill's Companions to the Christian Tradition 97 (Leiden: Brill, 2021):

(a) Eleanor J. GIRAUD, 'Dominican Chant and Liturgical Practices in the English Province', 343-69. (b) Nigel J. MORGAN, 'The Liturgical Manuscripts of the English Dominicans, ca.1250-ca.1530', 370-408.

30026. Ad fontes Tynecenses. Sakramentarz Tyniecki - perspektywa liturgiczno-muzyczna / Liturgical and musical perspectives on Tyniec Sacramentary, ed. Susi FERFOGLIA (Kraków: Tyniec Wydawnictwo Benedyktynów, 2020):

(a) Juan Pablo RUBIO SADIA, 'Muzyka w sakramentarzach: obecność i funkcje. Wprowadzenie do śpiewu celebransa' / 'La música en los sacramentarios: presencia y funcionalidad. Una aproximación al canto del celebrante', 219-46 / 249-76.

30027. Afinando ideas: aportaciones multidisciplinares de la joven musicología española, ed. Candela TORMO-VALPUESTA, Consuelo PÉREZ-COLODRERO (Granada: Editorial Universidad de Granada, 2020):

(a) Javier SASTRE GONZÁLEZ, 'Las grabaciones sonoras del Canto de la Sibila (19522017). Una aproximación desde la discología', 69-84. 
30028. Carta canta. Atti della giornata di studio. Pavia, Salone Teresiano 28 maggio 2019, ed. Marco D'AGOSTINO, Pier Luigi MULAS (Pavia: Univers Edizioni, 2020):

(a) Laura ALBIERO, 'Frammenti liturgici di reimpiego. Il caso di Pavia', 47-64. (b) Daniele SABAINO, Rodobaldo TIBALDI, 'Musica e liturgia nel frammento pavese pergamene sparse, scatola 1 bis', 65-92.

30029. Chanoines et chanoinesses des anciens Pays-Bas: Le chapitre de Maubeuge du IX au XVIII siècle, ed. Jean HEUCLIN, Christophe LEDUC, Histoire et civilisations (Villeneuve d'Ascq: Presse universitaires du Septentrion, 2019):

(a) Jean-François GOUDESENNE, ‘Un siècle avant Hildegarde et la réforme grégorienne, la "Vision" de sainte Aldegonde dans le paysage liturgique et musical du Hainaut et de la Gaule Belgique', 135-62.

30030. Die Beschneidung Jesu: Was sie Juden und Christen heute bedeutet, ed. Jan-Heiner TÜCK (Freiburg: Herder, 2020):

(a) Harald BUCHINGER, 'Die Feier der Beschneidung des Herrn am Oktavtag von Weihnachten: Liturgische Entwicklung und Entfaltung im ersten Jahrtausend', 147-85.

30031. Die Dynamik der Liturgie im Spiegel ihrer Bücher. Festschrift für Martin Klöckener / La dynamique de la liturgie au miroir de ses livres. Mélanges offerts à Martin Klöckener, ed. Hélène BRICOUT, Benedikt KRANEMANN, Davide PESENTI, Liturgiewissenschaftliche Quellen und Forschungen 110 (Münster: Aschendorff, 2020):

(a) Harald BUCHINGER, 'Zur Buchgeschichte und Liturgiegeschichte der Osterfeier in St. Gallen: Sakramentare im Kontext', 39-82. (b) Claire MAÎTRE, 'Les lectures médiévales des dimanches après la Pentecôte dans le domaine cistercien', 83-94. (c) Franz Karl PRASSL, 'Der Kantor und sein Dienst im Spiegel neuerer liturgischer Bücher', 561-80.

30032. Disiecta Membra Musicae: Studies in Musical Fragmentology, ed. Giovanni VARELLI, Studies in Manuscript Cultures 21 (Berlin/Boston: De Gruyter, 2020):

(a) Giovanni VARELLI, 'Preface', 1-6; (b) Susan RANKIN, 'Processional Chants in the Early Medieval Period: The Lesson of Fragments', 39-76; (c) David HILEY, 'Some Medieval Relics of Saints' Plainchant Offices', 77-94; (d) Jurij SNOJ, 'Music Fragments from Slovenia: Towards a Reconstruction of the Medieval Plainchant Manuscript Production', 97-115; (e) Sanna RANINEN, 'Make Do and Mend: Reworking Liturgical Parchment Manuscripts in Post-Reformation Sweden', 185-203; (f) Reinhard STROHM, 'A Collection of Fragments, or a Fragment of a Collection? The Musical Appendix of A-Wn Cod. 5094', 241-61; (g) Zsuzsa CZAGÁNY, 'Fragmenta Manuscriptorum Musicalium Hungariae Mediaevalis: From Traditional Methodologies Towards a Digital Corpus', 301-22.

30033. Glazba, Migracije i Europska Kultura. Svečani zbornik za Vjeru Katalinić. Music, Migration and European Culture. Essays in Honour of Vjera Katalinic, ed. Ivano CAVALLINI, Jolanta GUZI-PASIAK, Harry WHITE, Musicological proceedings 22 (Zagreb: Hrvatsko muzikološko društvo, 2020):

(a) Franz Karl PRASSL, “"Ex codicibus cisalpinis”: Das Kyriale von Peter Wagner (Graz 1904) und die Entstehung der Kyriale-Editionen in Solesmes 1883-1895-1905 als Vorbedingung für spätere Kontroversen um die Editio Vaticana', 351-75. 
30034. Global History, Visual Culture and Itineraries. Changes and Continuities, ed. Francisco José DÍAZ MARCILLA, Jorge TOMÁS GARCÍA, Yvette SOBRAL DOS SANTOS (Newcastle upon Tyne: Cambridge Scholars Publishing, 2021):

(a) Océane BOUDEAU, 'Introduction, Diffusion and Composition of Franco-Roman Plainsong in the Iberian Peninsula', 309-30.

30035. Guthlac: Crowland's Saint, ed. Jane ROBERTS, Alan THACKER (Donington: Shaun Tyas, 2020):

(a) Henry PARKES, 'Musical Portraits of St Guthlac', 277-97.

30036. Historiae. Liturgical Chant for Offices of the Saints in the Middle Ages, ed. David HILEY, Antiquae Musicae Libri 1 (Venezia: Fondazione Levi, 2021):

(a) Susan RANKIN, 'The Office in Carolingian hands', 3-32. (b) Henry PARKES, 'Theology and teleology in the festal Night Office: what performance directions reveal about the design and experience of historiae', 33-56. (c) Benjamin BRAND, 'Literary and musical borrowing in a versified office for St. Donatus of Arezzo', 57-72. (d) Harald BUCHINGER, 'On the hermeneutics and function of saints' offices: Observations and questions', 73-90. (e) Roman HANKELN, 'Music and text in saints' offices: two approaches', 91-134. (f) Nils Holger PETERSEN, 'Emotions and human identification in medieval saints' offices: a response to Roman Hankeln', 135-44. (g) Morne BEZUIDENHOUT, Mark BRAND, 'A web-based interface for the computational analysis and recognition of interval patterns in chants from late medieval saints' offices', 14564. (h) Kate HELSEN, 'Working with the research legacy of Andrew Hughes', 165-78. (i) Barbara HAGGH-HUGLO, 'Medieval offices from Ghent and Cambrai: some ways of interpreting their melodies', 179-222. (j) Jean-François GOUDESENNE, 'Geography and historiography in early West Frankish historiae (750-950)', 223-46. (k) Danette BRINK, "The seven historiae for the medieval cathedral of Trier: a conservative point of view', 247-60. (1) Robert KLUGSEDER, 'Saints' offices in Austria', 261-72. (m) Zsuzsa CZAGÁNY, 'Historiae in the Central European area: repertorial layers and transmission in Bohemia, Poland and Hungary', 273-96. (n) Jurij SNOJ, 'Late liturgical offices in Aquileian manuscripts', 297-312. (o) Marco GOZZI, 'Research on historiae in Italy: desiderata and opportunities', 315-32. (p) Cesarino RUINI, 'The offices of saints Adalbert, Hedwig and Stanislaus in Trent: a history of exclusion', 333-8. (q) Gionata BRUSA, Giulia GABRIELLI, 'Historiae in the South Tyrol: competing influences and historical developments in local chant composition', 339-72. (r) Stefania VITALE, 'The office of saint Eusebius of Vercelli from the eleventh to the twentieth century: prolegomena to an edition', 373-402.

30037. Into the Diaspora. Essays on Medieval and Early Modern Liturgical Music Manuscripts at the University of Sydney, ed. David ANDRES FERNÁNDEZ, Jane M. HARDIE, Wissenschaftliche Abhandlungen/Musicological Studies 113 (Kitchener, ON: Institute of Mediaeval Music, 2020):

(a) David ANDRÉS FERNÁNDEZ, 'Two Processionals from Female Monastic Houses (Fisher Library RB Add. Mss. 416 and 420)', 3-42. (b) Simon POLSON, 'Considering Two "Augustinian Antiphonals" (Fisher Library RB Add. Mss. 367 and 411)', 43-78. (c) Robert CURRY, 'Si tu hẹc servaveris, vitam eternam promito. A Seventeenth Century Personal Copy of the Clarist Rule and Constitutions from Peru (Fisher Library RB Add. Mss. 364)', 79-114. (d) Barbara SWANSON, 'Mary of the Snows, Paula the Widow, and Slavery to Mary in Fisher Library RB Add. Mss. 376: Defining Features of an Early Modern Compendium', 115-46. (e) Kathleen E. NELSON, 'Seeking Identity 
and History for an Antiphonal (Fisher Library RB Add. Mss. 413)', 147-85. (f) Santiago RUIZ TORRES, 'The Cantoral Fisher Library RB Add. Mss. 370: Musical and Liturgical Identity in the Spanish World', 187-234. (g) Grayson WAGSTAFF, 'Fisher Library RB Add. Mss. 377 and the Liturgy for the Dead: A Pre-Tridentine Survical in a Mid-Seventeenth-Century Book', 235-58. (h) Jane M. HARDIE, 'A Sibling for Salamanca? An Early Modern Graduale de Tempore (Fisher Library RB Add. Mss. 349) in Context', 259-304.

30038. Klang und Bedeutung. Diskurse über Musik. Zur Emeritierung von Joseph Willimann, ed. Juliane BRANDES, Moritz HEFFTER, Sarah PLATTE, Meinrad WALTER, Schriften der Hochschule für Musik Freiburg 9 (Hildesheim: Olms, 2021):

(a) Wulf ARLT, 'Materialgebrauch und Formgestaltung im "Neuen Lied" - Aspekte des einstimmigen Komponierens im 12. Jahrhundert', 1-26. (b) Jeremy LLEWELLYN, 'Sequences on Lake Constance or When Heinrich Isaac met Notker Balbulus', 231-66.

30039. Liturgia e cultura. Atti dell'XI Congresso Internazionale di Liturgia. Roma, Pontificio Ateneo sant'Anselmo - Pontificio Istituto Liturgico 9-11 maggio 2018, ed. Francesco BONOMO, Stefan GEIGER, Dominik JURCZAK, Fergus M.T. RYAN, Ecclesia Orans: Studi e Ricerche 2 (Napoli: Editrice Domenicana Italiana, 2019):

(a) Fergus M.T. RYAN, 'Music, Liturgy and Communication. Singing as Integral Part of the Liturgy', 71-84.

30040. Music and Instruments of the Middle Ages: Essays in Honour of Christopher Page, ed. Tess KNIGHTON, David SKINNER (Woodbridge: Boydell Press, 2020):

(a) John CALDWELL, 'The Development of the Latin Liturgical Psalter in England', 153-72.

30041. Musica Medieaeva liturgica III. Zbornîk príspevkov z muzikologickej konferencie konanej dna 6.-7- mája 2019, ed. Janka BEDNÁRIKOVÁ (Ruzomberok: Verbum, 2020):

(a) Adam SÝKORA, 'A few preliminary notes to codex Rkp. zv. 387 from a codicological and liturgical point of view', 28-48. (b) Rastislav LUTZ, 'Obiit dominus Fridericus burgravius. Zur Wanderung des Missale Rkp. 387', 55-61. (c) Eva VESELOVSKÁ, 'Die Notation des Missale Rkp. zv. 387 aus der Zentralbibliothek der Slowakischen Akademie der Wissenschaften', 71-9. (d) Csaba FRIGYES ORBÁN, Miklós István FÖLDVÁRY, 'Medieval Variants of the Mass Ordinary', 91-101. (e) Katalin SUBA, 'Researching the Occasional Rites of the Roman Liturgy: Recent Surveys of the Good Friday Services and their Old Latin Parallels', 138-59. (f) Piotr WIŚNIEWSKI, 'Palaeographic Characteristics of Musical Notation in the Twelfth-Century Pontificale Plocense', 171-80.

30042. Musik aufführen. Quellen - Fragen - Forschungsperspektiven, ed. Kai KÖPP, Thomas SEEDORF, Kompendien Musik 12 (Laaber: Laaber Verlag, 2020):

(a) Stefan MORENT, 'Aufführungspraxis in der Musik vor 1600 - Mangel an Quellen', 149-68.

30043. Musik im Zusammenhang: Festschrift Peter Revers zum 65. Geburtstag, ed. Klaus ARINGER, Christian UTZ, Thomas WOZONIG (Wien: Hollitzer, 2019):

(a) Franz Karl PRASSL, 'Der Salzburger Liber Ordinarius (1198) als musikhistorische Quelle', 133-55. (b) Stefan ENGELS, 'Die Handschrift Michaelbeuern A-MB Man. cart. 1 und die mensurierten Hymnen der monastischen Liturgie in Salzburg im 15. Jahrhundert', 157-75. 
30044. Respondámosle a concierto. Estudios en homenajes a Maricarmen Gómez Muntané, ed. Eduardo CARRERO, Sergi ZAUNER (Bellaterra: Institut d'Estudis Medievals - UAB, 2020):

(a) David ANDRÉS FERNÁNDEZ, Carmen MORTE GARCÍA, 'De manuscritos olvidados: los cantorales del antiguo monasterio de Santa Engracia de Zaragoza guardados en el Museo Arqueológico Nacional de Madrid', 43-64. (b) Juan Carlos ASENSIO, 'Ripoll \& Compostela: Arnaldo de Monte y el Codex Calixtinus', 65-76. (c) Jane M. HARDIE, 'Can the Pictures Tell the Story? Reflections on Three Sydney Medieval and Early Modern Liturgical Music Manuscripts', 139-50. (d) Kathleen NELSON, 'The Sacramentario de Sahagún and an Exultet', 185-98.

30045. Schaffen und Nachahmen. Kreative Prozesse im Mittelalter, ed. Volker LEPPIN, Samuel J. RAISER, Das Mittelalter. Perspektiven mediävistischer Forschung. Beihefte 16 (Berlin, Boston: De Gruyter, 2021):

(a) Stefan MORENT, 'Tradition und Wandel in der Überlieferungsgeschichte des Gregorianischen Chorals. Liturgisch-musikalische Fragmente und digitale Untersuchungsmethoden. Eine Einleitung zu den folgenden musikwissenschaftlichen Beiträgen', 527-9. (b) Waltraud GÖTZ, 'Einige Gründe für Variantenbildung im Gregorianischen Choral anhand von Beispielen aus dem Fragmentenbestand des Hauptstaatsarchivs Stuttgart', 545-50. (c) Stefan MORENT, 'Digitale Methoden zur Erforschung der Überlieferungsgeschichte des Gregorianischen Chorals', 551-4. (d) Paul HOPPE, Stefan MORENT, 'Computergestützte Tools zur Codierung des Gregorianischen Chorals. Ein neuer Eingabe-Editor für das MEI neumes-module', 555-60.

30046. Sounding the Past. Music as History and Memory, ed. Karl KÜGLE, Épitome musical (Turnhout: Brepols, 2020):

(a) Susan RANKIN, 'Introduction: Sung Histories', 37-44. (b) David EBEN, Susan RANKIN, 'Using the Past as Model: Musical Scripts in Books of the Prague Diocese', 75-100. (c) Pawel GANCARCZYK, 'Introduction: Tradition in the Musical Past', 11720. (d) Jan CIGLBAUER, 'From Tolerated Addition to Keepers of Tradition: The Authority of the "Past" in Latin Song in Central Europe in the Fourteenth and Fifteenth Centuries', 121-40. (e) Emanuel SIGNER, 'Tradition and Experimentation in Choirbooks Printed in Late Sixteenth and Seventeenth Century Italy', 157-90.

30047. The SAGE International Encyclopedia of Music and Culture, ed. Janet STURMAN (Thousand Oaks, CA: Sage, 2019):

(a) Eleanor J. GIRAUD, 'Chant', 496-500.

\section{Articles in periodicals and Festschriften}

30048. Laura ALBIERO, 'Reconstructing a Ninth-Century Sacramentary-Lectionary from Saint-Victor', Fragmentology, 3 (2020), 1-49.

30049. Rev. Ratislav ADAMKO, 'A Previously Unknown Repertoire of Alleluia Chants from the 1518 Graduale Cassoviense (Clmae 172a, 172b)', Roczniki Humanistyczne, 68/ 12 (2020), 25-44.

30050. David ANDRÉS FERNÁNDEZ, Carmen Julia GUTIÉRREZ, Emma HORNBY, Raquel ROJO CARRILLO, 'Processions and their Chants in the Old Hispanic Liturgy', Traditio, 75 (2020), 177-223. 
30051. David ANDRÉS FERNÁNDEZ, Cristina MARTÍ MARTÍNEZ, 'Hallazgos de fragmentos de canto gregoriano en Chile', Revista Musical Chilena, 71/227 (2017), 122-39.

30052. Janka BEDNÁRIKOVÁ, 'The Hymn Rex sanctorum angelorum in Notated Missal ms. 387 and its Partial Meaning in the Search of the Provenance of the Manuscript', Roczniki Humanistyczne, 68/12 (2020), 7-24.

30053. Océane BOUDEAU, 'Les livres liturgiques de la Chartreuse d'Évora (Portugal) témoins de la constitution d'un fonds cartusien et des changements dans la liturgie cartusienne à la fin du XVI ${ }^{\mathrm{e}}$ siècle', Acta musicologica, 92/2 (2020), 120-34.

30054. Océane BOUDEAU, Elsa DE LUCA, 'Erreur, variante et correction: 1'exemple du plainchant médiéval', Textus $\mathcal{E}$ Musica, 1 (2020). https://textus-et-musica.edel.univ-poitiers.fr / index.php?id=214.

30055. Océane BOUDEAU, Gaël SAINT-CRICQ, 'Les ressources et publications numériques de la musique médiévale', Revue de musicologie, 106/1 (2020), 175-90.

30056. Roger BOWERS, 'The "votive antiphon" among other sacred textual forms', Early Music, 48/2 (2020), 147-55.

30057. Laurent CALVIÉ, 'Un manuscrit médiéval d'anciens musicographes grecs: le Vaticano, BAV gr.2338', Scriptorium, 73/2 (2019), 219-50.

30058. David CATALUNYA, 'Ars subtilior in Organ Playing c. 1380-1420: Another Glimpse into a Late Medieval Unwritten Performance Practice', Kirchenmusikalisches Jahrbuch, 104 (2020), 113-43.

30059. Elsa DE LUCA, 'Aquitanian Notation in Iberia: Plainchant Fragments in Braga and Guimarães (11th-15th century)', Revue de Musicologie, 106/2 (2020), 329-72.

30060. Elsa DE LUCA, 'From Old Hispanic to Aquitanian Notation: Music Writing in Medieval Iberia', Anuario de Estudios Medievales, 50/2 (2020), 827-64.

30061. Litha EFTHYMIOU, Emma HORNBY, 'New Music Inspired by Old Hispanic Chant', Context, 45 (2019), 61-73.

30062. Ignasi M. FOSSAS COLET, Juan Pablo RUBIO SADIA, 'El ms. 880 de la Biblioteca de Montserrat: un breviari romanotoledà del segle $\mathrm{XIV}^{\prime}$, Miscel-lània litúrgica catalana, 27 (2019), 369-411.

30063. Giulia GABRIELLI, 'La musica nel regolamento scolastico della Collegiata di San Candido del 1614', Musica Docta. Rivista digitale di pedagogia e didattica della musica, 10 (2020), 15-23. https://musicadocta.unibo.it/issue/view/923.

30064. Giulia GABRIELLI, 'Gli inni nelle edizioni ambrosiane con musica del Seicento', Studi Gregoriani, 34 (2020), 9-27.

30065. M. Cecilia GAPOSCHKIN, 'Nivelon de Quierzy, the Cathedral of Soissons, and the Relics of 1205: Liturgy and Devotion in the Aftermath of the Fourth Crusade', Speculum, 95/4 (2020), 1087-129.

30066. Jean-François GOUDESENNE, 'La musique dans l'ancien diocèse de Thérouanne, de Guntbert de Saint-Bertin (831) à Drogon de Bergues (1070)', Annales du Comité Flamand de France, 70 (2019), 153-66. 
30067. Jean-François GOUDESENNE, 'La carte et le territoire du chant grégorien', Microscoop. Un regard sur les laboratoires en Centre Limousin Poitou-Charentes, 19 (2019), 24-5.

30068. Jean-François GOUDESENNE, Daniel-Odon HUREL, 'Redécouverte d'un mouvement monastique bénédictin pour la promotion et la sauvegarde des patrimoines liturgiques et musicaux d'Orient : historique et profils d'une musicologie innovante (1860-1930)', Revue des Traditions Musicales des mondes Arabes et Méditerranéen (RTM), 13 (2021), 85-102.

30069. James GRIER, 'De rebus incertis: Stephen of Liège and the Divine Office', Plainsong E Medieval Music, 29/2 (2020), 119-36.

30070. Miquel dels Sants GROS I PUJOL, 'L'oracional festiu hispànic de Silos (Londres, Brit. Lib., Add. 30852)', Miscel-lània litúrgica catalana, 27 (2019), 25-158.

30071. Carmen Julia GUTIÉRREZ, 'Librum de auratum conspice pinctum. Sobre la datación y la procedencia del Antifonario de León', Revista de Musicología, 43/1 (2020), 19-76.

30072. Carmen Julia GUTIÉRREZ, 'Francisco Franco y los reyes godos: la legitimación del poder usurpado por medio de la ceremonia y la música', Cuadernos de Música Iberoamericana, 33 (2020), Dosier: Música y ceremonial, 161-95.

30073. Karl D. HARTZELL, 'A fragment of a tenth-century English sacramentary', Anglo-Saxon England, 46 (2019), 259-70.

30074. Kristin HOEFENER, ‘Kultgeschichte als Musikgeschichte. Offizienzyklen zu Ehren der heiligen Ursula und der elftausend Kölner Jungfrauen unter besonderer Berücksichtigung des Laudeszyklus In choro sanctorum', Kirchenmusikalisches Jahrbuch, 103/104 (2019/2020), 85-103.

30075. Irene HOLZER, “"Das ist wol singen in gaÿstlicher fröd”. Musik der theologia mystica im Kloster Tegernsee', Kirchenmusikalisches Jahrbuch, 103/104 (2019/2020), 27-41.

30076. Emma HORNBY, Kati IHNAT, 'Continuous psalmody in the Old Hispanic liturgy', Scriptorium: revue internationale des études relatives aux manuscrits, 73/1 (2019), 1-33.

30077. Standley HOWELL, 'The emergence of a medieval pitch concept', Plainsong $\mathcal{E}$ Medieval Music, 29/2 (2020), 99-117.

30078. M. Ángel LÓPEZ, 'El officium de Cisneros: ¿origen hispánico o neomozárabe?', Cuadernos de Música Iberoamericana, 33 (2020), 225-40.

30079. M. Ángel LÓPEZ, 'Procedimientos de composición melódica en los officia y sacrificia neomozárabes', Revista de Musicología, 43/2 (2020), 501-28.

30080. Franz Karl PRASSL, 'Theology (en-) chanted: Christological aspects in the chants of the Triduum Sacrum', Cultura e Fede, 27/1 (2019), 25-34.

30081. Franz Karl PRASSL, ‘Kodeks Hartkera (CH-SGs 390/391) - genialna szkoła retoryki w służbie teologii liturgicznej [Der Codex Hartker (CH-SGs 390/391): Rhetorik und Rhythmische Artikulation als Ausdruck liturgischer Theologie]' (Übersetzung: Łukasz Wojtyczka), Pro Musica Sacra, 17 (2019), 43-61.

30082. Juan Pablo RUBIO SADIA, 'Consideraciones litúrgicas sobre el fragmento de brevario de la Biblioteca de Montserrat, ms. 1601 (Olim ms. 1117, folios de guarda)', Miscel-lània litúrgica catalana, 28 (2020), 115-38. 
30083. Ryan T. RUIZ, 'Sing for Us One of Zion's Songs: The Psalms and Eucharistic Anamnesis', Antiphon: A Journal for Liturgical Renewal, 24/3 (2020), 271-89.

30084. Daniel SAWICKI, 'The Development of the Melodic Formulas in Old Ruthenian Orthodox Church Monody on the Example of Dognaticon of the Second Tone Based on Selected 16th-18th Century West Ruthenian Irmologions', Roczniki Humanistyczne, 67/12 (2019), 39-57.

30085. Katarina ŠTER, 'A Chant Treatise in the Service of Two Monastic Traditions of the Modern Era: The Case of the Musices Choralis Medulla', Muzikološki zbornik/ Musicological Annual, 56/2 (2020), 153-81.

30086. Ana SUÁREZ GONZÁLEZ, Josep TORNÉ I CUBELLS, 'Cinc bifolis litúrgics medievals de l'Arxiu de la Basílica dels Sants Just i Pastor (Barcelona)', Miscel-lània litúrgica catalana, 28 (2020), 97-114.

30087. Haig UTIDJIAN, 'First session of the series of seminars Digital Humanities in Early Music Research: personal reflections', Hudební věda, 57/1 (2020), 83-7.

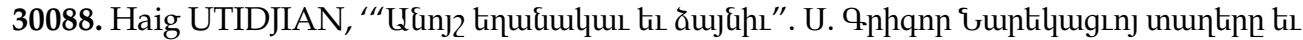

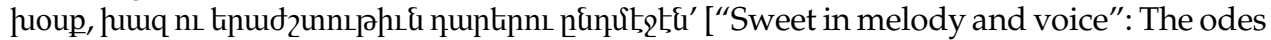
of St. Gregory of Narek through the centuries], Banber Matenadarani fuitinn

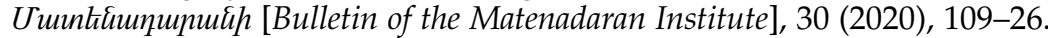

30089. Rose WALKER, 'Expressing Liturgical Change in Eleventh- and Twelfth-century Iberia through the Feast of the Holy Innocents', Anuario de Estudios Medievales, 50/2 (2020), 865-92.

30090. Rev. Piotr WIŚNIEWSKI, 'Melodies of the Prefaces Outside Mass in the 12th-Century Pontificale Plocense', Roczniki Humanistyczne, 67/12 (2019), 9-26.

30091. Rev. Piotr WIŚNIEWSKI, 'A Nineteenth-Century Processional from the Archive of the Bonifratres in Cracow (Kraków). A Contribution to Research into Latin Monody', Roczniki Humanistyczne, 68/12 (2020), 73-86.

\section{Ph.D. dissertations}

30092. Ángel Antonio CHIRINOS AMARO, 'Aspectos interpretativos del Códice musical de las Huelgas (E-BULH-11): un estudio en torno a la performance vocal del canto litúrgico bajomedieval' (Madrid: Universidad Complutense de Madrid, 2019).

30093. Kristin HOEFENER, ‘Untersuchungen zu Ursprung, Entwicklung und Verbreitung von Offizienzyklen zu Ehren der Heiligen Kölner Jungfrauen. Kulturgeschichte als Musikgeschichte' (Würzburg: Julius-Maximilian-Universität Würzburg, 2020).

30094. Patricia PELÁEZ BILBAO, 'Las secuencias del manuscrito Tortosa, Archivo Capitular, Cód. 135. Estudio y edición crítica', 2 vols. (Madrid: Universidad Complutense de Madrid, 2021).

\section{Websites and online databases}

30095. Catalogue des manuscrits notés en neumes français de la Bibliothèque nationale de France, http://manno.saprat.fr.

30096. Fragmentarium: Laboratory for Medieval Manuscript Fragments, https://fragmentarium.ms/. 
Ackermans, F. 30019a, 30020a

\section{Index of names}

Adamko, R. 30004, 30049

Agustoni, L. 30019a, 30020a

Albiero, L. 29003R, 30015a, 30016a, 30017f, 30028a, 30048

Altstatt, A. 30017am

Andreoni, F. 30023d

Andrés Fernández, D. 30037, 30037a, 30044a, 30050, 30051

Aringer, K. 30043

Arlt, W. 30038a

Asensio, J.C. 30044b

Aubert, E.H. 30021b

Bailey, T. 30022c

Bain, J. 30017t

Batoff, M. 30017al

Bednáriková, J. 30004, 30041, 30052

Behrendt, I. 30017t, 30019a, 30020a, 30020d

Bernhard, M. 30001

Betteray, D.v. 30017aa

Bezuidenhout, M. 30017z, 30036g

Bonomo, F. 30039

Borders, J. 30017, 30017c, 30017m

Boudeau, O. 30017aj, 30021c, 30034a, 30053, 30054, 30055

Bowers, R. 30056

Brand, B. 30036c

Brand, M. 30036g

Brandes, J. 30038

Bricout, H. 30031

Brink, D. 30017ac, 30036k

Brusa, G. 30002, 30023b, 30036q

Buchinger, H. 30030a, 30031a, 30036d

Calabretto, R. 30017b

Caldwell, J. 30040a

Calloni, J.M. 30022d

Calvié, L. 30057

Carrero, E. 30044

Catalunya, D. 30017h, 30058

Cavallini, I. 30033

Chirinos Amaro, A.A. 30092

Ciglbauer, J. 30045d

Colantuono, M.I. 27/28024R

Croff, D. 30017a

Csomó, O. 30017ap

Curry, R. 30037c

Cyrus, C.J. 27/28027R

Czagány, Z. 30032g, 30036m

D'Agostino, M. 30028

De Luca, E. 30017g, 30054, 30059, 30060

Denoël, C. 30015 
Díaz Marcilla, F.J. 30034

Dyer, J. 30001R, 30021a

Eben, D. 30046b

Efthymiou, L. 30061

Engels, S. 30043b

Ermini Pani, L. 30016

Ferfoglia, S. 30026

Ferreira, M.P. 30017ao

Fischer, R. 30019a, 30020a

Földváry, M.I. 30041d

Fossas Colet, I.M. 30062

Frigyes Orbán, C. 30041d

Gabrielli, G. 30017v, 30023a, 30036q

Gagliano, L. 30024a

Gancarczyk, P. 30046c, 30063, 30064

Gaposchkin, M.C. 30065

Garajová, V. 30005

Garzetti, M. 30022e

Gattozzi, B. 30017at

Geiger, S. 30039

Giraud, E.J. 30025, 30025a, 30047a

Göschl, J.B. 30019a, 30020a

Götz, W. 30045b

Goudesenne, J.F. 30017ai, 30029a, 30036j, 30066, 30067, 30068

Gozzi, M. 30017i, 30022f, 300360

Grier, J. 30069

Gros i Pujol, M.d.S. 30070

Gruber, G. 30006

Gutierrez, C.J. 30050, 30071, 30072

Guzi-Pasiak, J. 30033

Haggh-Huglo, B. $30036 \mathrm{i}$

Hankeln, R. 30017ab, 30036e

Hardie, J.M. 30037, 30037h, 30044c

Hartzell, K.F. 30073

Haug, A. 30017p

Heffter, M. 30038

Helsen, K. 30017t, 30036h

Herrmann-Schneider, H. 30007

Heuclin, J. 30029

Hild, E.S. 30017r

Hiley, D. 30032c, 30036

Hoefener, K. 30074, 30093

Holzer, I. 30075

Hönerlage, C. 30008, 30019a, 30020a

Hoppe, P. 30045d

Hornby, E. 30050, 30061, 30076

Howell, S. 30077

Høye, M.J. 30017w

Hughes, A. 30036h, 
Hurel, D.O. 30068

Ihnat, K. 30076

Janke, A. 29014R.

Joppich, G. 30019c

Jurczak, D. 30039

Kelly, T.F. 30017aq, 30022g

Kiss, G. 30017u

Klarer, S. 30018d

Klugseder, R. 300361

Knighton, T. 30040

Koch, L. 30019a, 30020a

Kohlhäufl, J. 30019a, 30020a

Koláček, J. 30017s

Kranemann, B. 30031

Kubieniec, J. 30023e

Kügle, K. 30046

Kujumdzieva, S. 30017au

Lacoste, D. 30017s

Lagergren, K.S. 30017j

Leduc, C. 30029

Leigh-Choate, T. 30017ak

Leppin, V. 30045

Levey, M.T. 30009

Linde, C. 30025

Livljanić, K. 30017aq

Llewellyn, J. 30038b

Long, C. 30021d

Long, S.A. 30017x

López, M.Á. 30078, 30079

Lutz, R. 30004, 30041b

Magnoli, C. 30022b

Mailänder, R. 30010

Maître, C. 30031b

Malaspina, S.M. 30024a

Maloy, R. 30011

Markham, E.J. 30017az

Martí Martínez, C. 30051

Metz, S. 30019a, 30020a

Miklós, R. 30017o

Milanese, G. 30022, 30022a, 30023, 30024

Morandi, N. 30017d, 30017af

Morent, S. 30012, 30018e, 30042a, 30045a, 30045c, 30045d

Morgan, N.J. 30025

Morte García, C. 30044a

Mulas, P.L. 30028

Müller, W.W. 30018

Nardini, L. 27/28003R, 30017ar

Nelson, K.E. 30037e, 30044d 
Nikolakopoulos, K. 30018c

Nishiwaki, I. 30020c

Parkes, H. 30035a, 30036b

Peattie, M. 30017as

Peláez Bilbao, P. 30094

Pérez-Colodrero, C. 30027

Perrin, X. 30021e

Pesenti, D. 30031

Petersen, N.H. $30036 \mathrm{f}$

Pfisterer, A. 30017e

Platte, S. 30038

Poliakova, S. 30017ay

Polson, S. 30037b

Prassl, F.K. 27/28016R, 30018b, 30020b, 30031c, 30033a, 30043a, 30080, 30081

Raiser, S.J. 30045

Raninen, S. 30032e

Rankin, S. 30032b, 30036a, 30046a, 30046b

Ricossa, L. 30023c

Riemann, H. 30020d

Roberts, J. 30035

Rojo Carrillo, R. 30013, 30017ad, 30050

Roncroffi, S. 30014, 30017ag

Rösler, D. 30019a, 30020a

Rubio Sadia, J.P. 30017k, 30026a, 30062, 30082

Ruini, C. 30036p

Ruiz, R.T. 30083

Ruiz Torres, S. 30017k, 30037f

Rumphorst, H. 30019a, 30019d, 30020a

Rusconi, A. 30022, 30022b, 30022h, 30023, 30024

Ryan, F.M.T. 30039, 30039a

Sabaino, D. 30028b

Sachs, K.J. 30001

Saint-Cricq, G. 30055

Sandmeier, R. 300171

Sastre González, J. 30027a

Saulnier, D. 27/28024R

Sawicki, D. 30084

Seedorf, T. 30042

Signer, E. 30045e

Siri, F. 30015

Skinner, D. 30040

Snoj, J. 30017n, 30032d, 30036n

Sobral dos Santos, Y. 30034

Šter, K. 30085

Stingl jun., A. 30019a, 30019b, 30020a

Stirpe, F. 30024c

Strohm, R. $30032 \mathrm{f}$

Sturman, J. 30047

Suárez González, A. 30086 
Suba, K. 30041e

Swanson, B. 30037d

Sýkora, A. 30041a

Tessari, S. 30017av

Thacker, A. 30035

Tibaldi, R. 30028b

Tomás García, J. 30034

Tormo-Valpuesta, C. 30027

Torné i Cubells, J. 30086

Toigo, D. 30017ah

Tück, J.H. 30030

Urberg, M. 30017an

Utidjian, H. 30087, 30088

Utz, C. 30043

Valli, N. 30024b

Varelli, G. 30032, 30032a

Veselovská, E. 30004, 30041c

Vitale, S. 30036r

Vlhová-Wörner, H. 30003, 30017y

Voigt, K. 30017q

Wagner, F. 30018, 30018a

Wagstaff, G. 30037g

Walker, R. 30089

Walter, M. 30038

Wanek, N.M. 30017ax

White, H. 30033

Winkelmüller-Urechia, M. 29017R.

Wiśniewski, P. 30041f, 30090, 30091

Wolfram, G. 30017aw

Wozonig, T. 30043

Zahradniková, Z. 30004

Zanoncelli, L. 30017b

Zauner, S. 30044

Zippe, S. 30019a, 30020a

Zühlke, H. 30017ae

Agnus Dei 30017y

\section{Index of subjects}

Alleluia 30017u, 30049

Amalar of Metz 30020c

Ambrosian chant 30022, 30023, 30024, 30064

Antiphonary 29003R, 30017n, 30017aq, 30022d, 30022f, 30037b, 30037e, 30044a, 30071

Antiphons 30022c, 30024b, 30024c, 30056

Antiquity 30006, 30012, 30018

Arnaldo de Monte 30044b

Augustins 30037b

Australia 30037, 30044c

Austria 30007, 30017o, 30017w, 300361, 30043a, 30043b 
Belgium 30029a, 30036i

Benedictine 30017ae, 30067

Beneventan chant 30017aq, 30017ar, 30017at

Bohemia see Czech republic

Breviary 30023e, 30062, 30082

Byzantine chant 30017av, 30017aw, 30017ax, 30018c, 30057

Cantional 30003

Canto de la Sibila 30027

Cantor 30031c

Cantoral 30037f, 30044a

Carolingians 30036a

Catalogue 30005

Cantus planus Study Group 30017

Cartusians 30053

Centonisation (see Formulas) 30008

Chile 30051

Christmas 30020c, 30030a

Cistercians 30007, 30031b

Clarists 30037c

Codicology 30017h

Concile 30018e

Credo 30022h

Croatia 30017ap, 30033b

Crusade 30065

Czech Republic 30003, 30017y, 30036m, 30046b

Databases 30017s

Digital Humanities/Computer-assisted research 30017r, 30017t, 30017z, 30032g, 30036g, 30045a, 30045b, 30045c, 30045d, 30087

Dognaticon 30084

Dominican chant 27/28027R, 30025

Doxology 30017aw

Drogon de Bergues 30066

Early medieval period $30032 \mathrm{~b}$

Easter 30031a, 30080

Editio vaticana 30033 a

Education 30063

Europe 30017l, 30017u, 30018e, 30033

Exultet 30044d

Formula 30008, 30017aa, 30017ar, 30084

Fragments 29014R, 30005, 30017k, 30018e, 30022d, 30028, 30032, 30045a, 30045b, 30051, 30082, 30086, 30096

France 30015, 30017f, 30017h, 30017m, 30017x, 30017ai, 30017aj, 30017ak, 30021b, 30029a, 30033a, 30036i, 30048, 30059, 30065, 30066

Franco, Francisco 30072

Franks 30036j

Germany 27/28027R, 29014R, 30001, 30002, 30017w, 30017ac, 30017ae, 30017al, 30017am, 30036k, 30038b, 30045b, 30074, 30075, 30093

Gradual (chant book) 30019a, 30020a, 30037h, 30049 
Graduals (chants) 30008

Great Britain 30015, 30025, 30035, 30040a, 30072

Gregorian Chant 27/28024R, 29017R, 30006, 30008, 30010, 30012, 30018d, 30019, 30020, 30021d, 30024c, 30033a, 30033b, 30034a, 30045a, 30045d, 30051, 30054, 30067

Guntbert de Saint-Bertin 30066

Guy de Saint-Denis 27/28003R

Hagiography 30017ac, 30017af, 30017ag, 30017ak, 30017am, 30017ap, 30021d, 30036

Hainaut 30029a

Hildegard 30029a

Hispanic rite 30011, 30013, 30017ad, 30050, 30061, 30071, 30076, 30089

Historiae 30036

Holland 30017j

Hungary 29014R, 30032g, 30036m

Hymn 30017at, 30043b, 30052

Illumination $27 / 28027 \mathrm{R}, 30044 \mathrm{c}$

Instruments 30040, 30058

Isaac, Heinrich 30038b

Italy 30014, 30016a, 30017f, 30017i, 30017m, 30017ae, 30017af, 30017ag, 30017ah, 30017ai, 30017aq, 30022f, 30023b, 30024a, 30036n, 30036o, 30036p, 30046e

Kyriale 30009, 30033a

Kyrie 30017w

Language 30017ab

Laudes 30074

Lectionary 30048

Liber ordinarius 30002, 30017o, 30043a

Liquescence 30019c

Liturgical manuscripts $30025 \mathrm{~b}$

Liturgy 27/28027R, 29017R, 30008, 30009, 30010, 30013, 30015a, 30016a, 30017i, 30017k 30017o, 30017ah, 30017ai, 30017ap, 30018e, 30023b, 30028b, 30024b, 30025a, 30025b, 30030a, 30031, 30037, 30039, 30041a, 30041e, 30043b, 30050, 30053, 30065, 30076, 30082,30089

Manuscripts 30014, 30015, 30016a, 30017i, 30017j, 30017l, 30017w, 30017ag, 30017ar, 30017at, 30017av, 30019d, 30025a, 30031a, 30032d, 30036n, 30037, 30055, 30095

Mass 30017ar, 30019a, 30083

Missale 30004, 30041, 30052

Moravia see Czech republic

Music theory 27/28003R, 30001

Mystics 30075

Neo-Mozarabic rite 30078, 30079

Neumes 30017e, 30017t, 30017as, 30020d, 30021e

New Latin song 30017h

New Philology 30017r

Nivelon de Quierzy 30065

Notation (see also Neumes) 30017e, 30017f, 30017g, 30017as, 30021b, 30022g, 30024c, 30041c, 30046b, 30059, 30060, 30077

Notker Balbulus 30038b

Nova Cantica 30038a 
Odes 30088

Offertory 30019b, 30019d

Office 30017aa, 30017af, 30017ak, 30017am, 30017aq, 30021d, 30035a, 30036, 30069, 30074, $30078,30079,30093$

Old Roman chant 29017R, 30021a

Orational 30070

Ordinarium Missae 30009, 30017x, 30041d

Orient 30067

Patrology 30008

Pavia 30028

Pentecost 30031b

Performance practice 30031c, 30042a, 30046, 30092

Peru 30037c

Poland 30023e, 30026, 30036m, 30090, 30091

Pontificale Romanum $30017 \mathrm{~m}$

Pontificale Plocense 30041f, 30090

Portugal 30017ao, 30034a, 30053, 30059, 30060, 30089

Prefaces 30090

Processions 30017an, 30022c, 30032b, 30050

Processionals 30024c, 30037, 30044c, 30091

Psalms 30083

Psalmody 30076

Psalter 30040a

Psalterium 27/28016R

Relics 30065

Relics of Saints' offices 30032c

Responsories 30017ab, 30024b

Rhetoric 30020b, 30081

Rhythm 30020b, 30020d, 30081

Russia 30017ay

Ruthenia 30084

Sacramentary 30026, 30031a, 30044d, 30048, 30072

Sacrifice 30011

Saints (also see Hagiography)

Adalbert 30036p

Aldegonde 30029a

Donatus of Arezzo 30036c

Cecilia 30017af

Eusebius of Vercelli 30036r

Gregory of Narek 30088

Guthlac 30035a

Hedwig 30036p

Mary of the Snow 30037d

Paula the Widow 30037d

Stanislaus 30036p

Ursula 30074

Virgin Mary 30017ap, 30037d

Sanctoral 30017aj

Scandinavia 30004 
Semiotics 30017p

Semiology (Gregorian) 30019c

Sequences 30038b, 30094

Slovakia 30004, 30005, 30041

Slovenia 30017n, 30032d

Song of the Sibyl 30027a

South Africa 300171

Spain 30012, 30013, 30017k, 30027, 30034a, 30037f, 30037h, 30044a, 30044b, 30044d, 30062, 30070, 30071, 30086, 30089

Stephen of Liège 30069

Sticheraria 30017ay

Sweden 30017an, 30032e

Switzerland 30018d, 30018e, 30020d, 30031a

Theology 30010, 30018, 30020b, 30036b, 30080, 30081, 30083

Theory 27/28003R, 30057, 30077, 30085

Transcription 30017p

Transmission 30054, 30058

Tropes 30017v

Tropologion 30017au

Tyrol 30007, 30017v, 30036q, 30063

Venice 30017ao, 30017av

Versus 30017q, 30022e

Vespertinus 30013, 30017ad

Visitatio sepulchri 30017al

Wagner, Peter 30033a

Antiphoner of Izola 30017an

\section{Index of manuscripts}

Bassano del Grappa, Civic Library gr. 34B19 30017av

Bratislava, Ústredna knižnica Slovenskej akadémie vied Rkp. zv. 387 30041, 30052

Budapest, National Széchényi Library, Clmae 172a/172b 30049

Codex Calixtinus 30044b

Codex Hartker 30017aa, 30020b, 30081

Codex Hartker St. Gallen 390/391 30019c

Codex Las Huelgas 30092

Fragments (see list in Index of Subjects, Fragments)

Graz, Universitätsbibliothek $156630032 \mathrm{f}$

Kraków, National Library Rps BOZ 8 (Tyniec Sacramentary) 30026

León, Archivo Catedralicio núm. 830071

London, British Library, Add. MS. 3085230070

Madrid, Museo Arqueológico Nacional, 1869/23/102, 103 and 104 30044a

Michelbeuern, Man. Cart. 1 30043b

Montserrat, Biblioteca de Montserrat, ms. 88030062

Montserrat, Biblioteca de Montserrat, ms. 160130082

Oxford, Bodleian Library, MS. Canonici Liturgical $37530017 \mathrm{~m}$

Paris, Bibliothèque nationale, fonds latin 1139 30017h, 30017q

Sacramentario de Sahagún, Madrid, BNE, VITR/20/8, 30044d

Sydney, Fisher RB Add. 349 30037h

Sydney, Fisher RB Add. 364 30037c 
Sydney, Fisher RB Add. 367 30037b

Sydney, Fisher RB Add. $37030037 f$

Sydney, Fisher RB Add. 376 30037d

Sydney, Fisher RB Add. 377 30037g

Sydney, Fisher RB Add. 411 30037b

Sydney, Fisher RB Add. 413 30037e

Sydney, Fisher RB Add. 416 30037a

Sydney, Fisher RB Add. 420 30037a

Tortosa, Archivo Capitular, Cód. 13530094

Trento, Biblioteca del Castello di Buonconsiglio, Fondo Feininger, ms. FC $7430022 \mathrm{f}$

Vatican City, Biblioteca Vaticana, Greacus 771 30017au

Vatican City, Biblioteca Vaticana, Greacus 233830057

Vaticanus Reginensis Latinus 334 30024c 